\title{
Rapid Detection of Sepsis using CESDA: the Caenorhabditis elegans Sepsis Detection Assay
}

\author{
Ling Fei Tee ${ }^{[1]}$, Toh Leong Tan ${ }^{[2]}$, Hui-min Neoh ${ }^{[1]}$ and Rahman Jamal[1] \\ [1]. UKM Medical Molecular Biology Institute (UMBI), Universiti Kebangsaan Malaysia, Malaysia. \\ [2]. Department of Emergency Medicine, Faculty of Medicine, Universiti Kebangsaan Malaysia, Malaysia.
}

\begin{abstract}
Introduction: The nematode Caenorhabditis elegans was used as a biological sensor to detect the urine of sepsis patients (CESDA assay). Methods: C. elegans was aliquoted onto the center of assay plates and allowed to migrate towards sepsis (T) or control (C) urine samples spotted on the same plate. The number of worms found in either (T) or (C) was scored at 10-minute intervals over a 60-minute period. Results: The worms were able to identify the urine ( $<48$ hours) of sepsis patients rapidly within 20 minutes (AUROC $=0.67, p=0.012$ ) and infection within 40 minutes (AUROC $=0.80, \mathrm{p}=0.016$ ). Conclusions: $\mathrm{CESDA}$ could be further explored for sepsis diagnosis.
\end{abstract}

Keywords: Sepsis. C. elegans. Chemotaxis assay. C. elegans Sepsis Detection Assay (CESDA).

Sepsis is a condition in which patients develop lifethreatening single or multi-organ dysfunction due to dysregulated host response to infection ${ }^{1}$. Even today, the diagnosis of sepsis remains a challenge, as there is no single reliable test for its early confirmation or exclusion. Blood cultures offer low sensitivity, viral serology tests are costly, while common laboratory screening parameters of white blood cell counts, erythrocyte sedimentation rate, and C-reactive protein have both poor sensitivity and specificity for diagnosing sepsis ${ }^{2-6}$. Early sepsis diagnosis is important as it can help emergency medicine physicians perform risk stratification and initiate antibiotics promptly (if required), leading to better patient management and outcome ${ }^{7}$.

Caenorhabditis elegans is a nematode widely used for studies in developmental biology and is a model organism for many diseases, particularly in neurobiology ${ }^{8}$. Recently, the nematode was reported to be able to sense and differentiate human cancer cell secretions, cancer tissues and urine from healthy control samples 9 . The chemotaxis assay, designated as the $C$. elegans nematode scent detection test (NSDT) works best on urine samples, with a sensitivity of $95.8 \%$ and specificity of

Corresponding author: Toh Leong Tan, MD / Hui-min Neoh, Ph.D., e-mail: sebastiantt1@yahoo.co.uk / hui-min@ppukm.ukm.edu.my ORCID: 0000-0002-6771-0141

Received 19 July 2018

Accepted 31 August 2018
$95.0 \%$. The positive predictive value and efficiency of the test were $67.6 \%$ and $95.0 \%$, respectively.

It has been reported that cancer is the most common comorbidity associated with infection, and they share multiple similarities $^{10-12}$. For example, inflammatory processes mediated by $\mathrm{T}$ cells are expressed in both diseases. Persistent immune activation and inflammation lead to the activation of common signaling pathways that regulate immunity in both cancer and infection. In addition, inflammatory processes in both diseases lead to the release of similar pro-inflammatory and antiinflammatory cytokines, increased levels of reactive oxygen and nitrogen species, tissue wasting, and increased apoptosis ${ }^{12}$.

Although the olfactory molecule emitted from cancer urine samples that were sensed by the worms is still unknown, we suspect that the inflammatory process during infection will also lead to the emission of specific olfactory molecules that could be detected by $C$. elegans in urine samples of patients with infection, and perhaps, sepsis.

To test this hypothesis, we proceeded to perform a C. elegans chemotaxis assay (designated as the C. elegans Sepsis Detection Assay [CESDA]) and evaluated the sensitivity and specificity of CESDA in detecting sepsis using urine samples obtained from patients admitted to the Department of Emergency Medicine (ED) of Universiti Kebangsaan Malaysia Medical Centre (UKMMC). The study was designed as a pilot, proof-of-concept study carried out from January 2016 until June 2016 with the ethics reference number: UKM PPI/111/8/JEP-2016-060. All 
procedures for the study were performed in accordance with ethical standards of the Helsinki Declaration of 1975, as revised in $2000^{5}$.

During the study period, a total of 166 patients aged 18 years or older were admitted to the ED-UKMMC for suspected sepsis and infection. Sepsis was diagnosed according to the 2001 SCCM/ESICM/ACCP/ATS/SIS International Sepsis Definitions Conference criteria ${ }^{13}$, in which sepsis is defined as a condition where patients have a minimum of two systemic inflammatory response syndrome (SIRS) criteria together with suspected infection. Patients with infection without the SIRS criteria were classified as having infection only. Confirmatory diagnosis of sepsis samples was determined using a combination of clinical criteria assessment and standard laboratory protocols.

Out of the 166 patients, 56 subjects consented to the study and provided urine samples with written informed consent. Eleven patients were then excluded from the study: four had autoimmune diseases, two had anaphylaxis, two already initiated antibiotics, one had malignancy and two were on long-term steroid medication. The remaining 45 patients were eligible for this study. Among these, 36 patients' urine samples were collected and tested in less than 24 hours, while the remaining nine samples were collected and assayed between 24 and 48 hours. Forty-five control samples were obtained from healthy subjects who, at the time of the study, were free from infection or cancer.

Approximately $5 \mathrm{ml}$ of urine was collected from each study subject using a sterile urine container and stored at $4^{\circ} \mathrm{C}$ for not more than 48 hours until CESDA was performed. The assay was conducted as described by Hirotsu et al. with some modifications ${ }^{9}$. Briefly, urine samples were pre-warmed to room temperature before spotting onto assay plates. Spotting design for samples of this study was slightly different from the NSDT assay described by Hirotsu et al. CESDA plates were divided into four quadrants, with test (sepsis, T) and control (C) samples spotted onto two different quadrants, respectively. In addition, spots, where urine samples were dispensed on the agar, were also marked as points in the respective quadrants (Figure 1). In contrast, the NSDT assay plate only had two quadrants (one each for $\mathrm{T}$ and $\mathrm{C}$, respectively), and four urine dispense points.

Ten microliters of urine samples were spotted onto each point on the CESDA assay plate. Following that, $2 \mu \mathrm{L}$ of wildtype C. elegans $\mathrm{N} 2$ worms (about 50 worms) were transferred from a maintenance plate, washed with M9 buffer and aliquoted onto a circle located at the center of the assay plate (Figure 1). Worms were then allowed to migrate on the plate for 60 minutes. The number of worms found in the location of both test and control quadrants as well as points was observed using a stereomicroscope and recorded for each 10 minute-interval for 60 minutes.

The CESDA index of worms for each sample was then calculated as below: Number of worms in test quadrant/point - Number of
worms in control quadrant/point

Total number of worms (Test + Control)

Where a CESDA index near +1 represented attraction of worms towards the sample, while a CESDA index near -1 signified repulsion ${ }^{14}$. The correlation of positive/negative chemotaxis index with sepsis/control samples was determined via Pearson's chisquare test, where $p<0.05$ was considered statistically significant.

Interestingly, our results showed that the CESDA assay could differentiate sepsis patients from healthy controls and also from patients who had only infection. CESDA index calculated using worm numbers found on points rather than in quadrants was associated more strongly with sepsis and infection (Table 1).

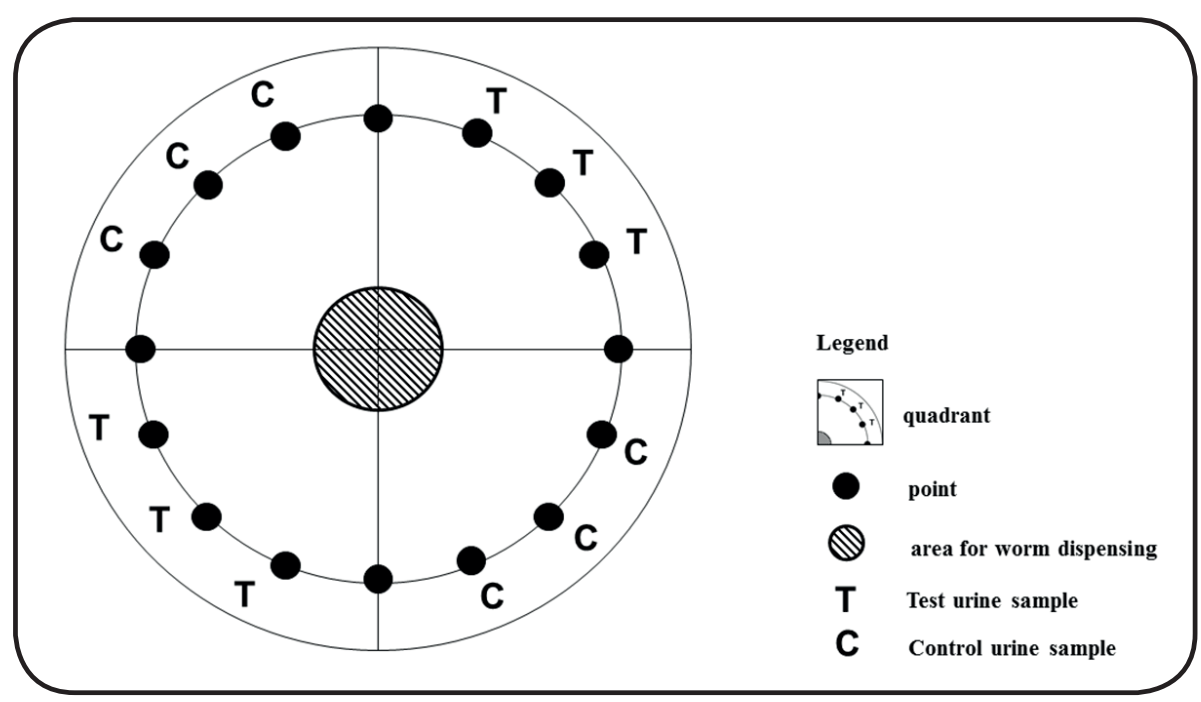

FIGURE 1: Plate design for CESDA. Urine samples were dispensed onto points (black-colored full circles either labeled as 'T' (sepsis) or 'C' (control). Worms were then transferred onto a circle located in the middle of the assay plate and allowed to migrate. The number of worms (located in either ' $\mathrm{T}$ ' or ' $\mathrm{C}$ ' quadrants or at the exact points) was scored for each 10 minute-interval in the 60-minute assay. 
All patients who were diagnosed with sepsis were found to have a CESDA index of $>0.1$ at $20 \mathrm{~min}$ (positive predictive value [PPV] $=87 \%$ for samples collected between 24 and 48 hours, and PPV $=92 \%$ for samples collected in less than 24 hours). Patients who were categorized as having infection without sepsis had a CESDA index of $>0.232$ at $40 \mathrm{~min}$ ( $\mathrm{PPV}=92 \%$ for samples collected between 24 and 48 hours, and PPV $=95.8 \%$ for samples collected in less than 24 hours) (Table 2). On further analysis, we found that CESDA could differentiate the urine of sepsis patients from controls as early as 20 minutes $(p=0.012)$. The accuracy of the CESDA index was lower for samples which were collected and tested in 24 hours compared with those collected between 24 and 48 hours (Table 2). The ability of CESDA to predict both sepsis and infection (for assays based on points) is shown in Table 2.
Our chemotaxis assay was a modification of the NSDT

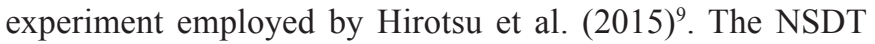
method was published in 2015 and the main finding was that it was able to differentiate the urine of 24 cancer patients from those of 218 healthy subjects. The authors reported that five healthy subjects, who had been previously identified as having 'cancer' according to the NSDT, received a cancer diagnosis 2 years after the time of the study. The NSDT was found to be robust for predicting both various types and staging of cancer, and has been suggested to be used as a cancer screening test ${ }^{9}$.

In our study, the CESDA index was calculated using worm counts at points (our study) rather than quadrants on the assay plate (as in the NSDT protocol) and provided a stronger association of the index with both infection and sepsis. Stronger

TABLE 1: CESDA index and AUROC values for urine samples collected and assayed in 24 hours ( $n=36)$ and 48 hours ( $\mathrm{n}=45$ ).

CESDA Index and AUROC

\begin{tabular}{|c|c|c|c|c|c|c|c|c|}
\hline \multirow{3}{*}{$\begin{array}{l}\text { Worm } \\
\text { location, } \\
\text { time }\end{array}$} & \multicolumn{4}{|c|}{ Sepsis } & \multicolumn{4}{|c|}{ Infection } \\
\hline & \multicolumn{2}{|c|}{ Median \pm SD } & \multicolumn{2}{|c|}{ AUROC ${ }^{a}(95 \% \mathrm{Cl})$} & \multicolumn{2}{|c|}{ Median \pm SD } & \multicolumn{2}{|c|}{ AUROC $(95 \% \mathrm{Cl})$} \\
\hline & 24 hours & 48 hours & 24 hours & 48 hours & 24 hours & 48 hours & 24 hours & 48 hours \\
\hline $\begin{array}{l}\text { Quadrant, } \\
10 \text { min }\end{array}$ & $0.095 \pm 0.271$ & $0.098 \pm 0.256$ & $0.62(0.41-0.84)$ & $0.60(0.44-0.77)$ & $0.095 \pm 0.272$ & $0.095 \pm 0.254$ & $0.57(0.38-0.77)$ & $0.66(0.49-0.82)$ \\
\hline $\begin{array}{l}\text { Quadrant, } \\
20 \text { min }\end{array}$ & $0.053 \pm 0.204$ & $0.076 \pm 0.206$ & $0.65(0.39-0.92)$ & $0.59(0.41-0.78)$ & $0.037 \pm 0.227$ & $0.053 \pm 0.221$ & $0.60(0.37-0.83)$ & $0.62(0.43-0.81)$ \\
\hline $\begin{array}{l}\text { Quadrant, } \\
30 \text { min }\end{array}$ & $0.123 \pm 0.250$ & $0.133 \pm 0.249$ & $0.56(0.32-0.79)$ & $0.62(0.47-0.78)$ & $0.0133 \pm 0.258$ & $0.123 \pm 0.251$ & $0.65(0.47-0.83)$ & $0.59(0.41-0.77)$ \\
\hline $\begin{array}{l}\text { Quadrant, } \\
40 \text { min }\end{array}$ & $0.123 \pm 0.243$ & $0.132 \pm 0.242$ & $0.53(0.28-0.78)$ & $0.64(0.43-0.84)$ & $0.137 \pm 0.2445$ & $0.123 \pm 0.239$ & $0.70(0.46-0.95)$ & $0.55(0.37-0.74)$ \\
\hline $\begin{array}{l}\text { Quadrant, } \\
50 \text { min }\end{array}$ & $0.179 \pm 0.242$ & $0.140 \pm 0.254$ & $0.62(0.35-0.89)$ & $0.61(0.41-0.81)$ & $0.180 \pm 0.266$ & $0.137 \pm 0.278$ & $0.78(0.62-0.95)$ & $0.60(0.40-0.81)$ \\
\hline $\begin{array}{l}\text { Quadrant, } \\
60 \text { min }\end{array}$ & $0.207 \pm 0.250$ & $0.197 \pm 0.281$ & $0.59(0.32-0.87)$ & $0.61(0.43-0.79)$ & $0.208 \pm 0.290$ & $0.194 \pm 0.304$ & $0.73(0.52-0.93)$ & $0.58(0.38-0.77)$ \\
\hline $\begin{array}{l}\text { Point, } \\
10 \text { min }\end{array}$ & $0.143 \pm 0.508$ & $0.219 \pm 0.498$ & $0.52(0.30-0.74)$ & $0.45(0.26-0.64)$ & $0.165 \pm 0.510$ & $0.224 \pm 0.494$ & $0.55(0.35-0.75)$ & $0.45(0.23-0.67)$ \\
\hline $\begin{array}{l}\text { Point, } \\
20 \text { min }\end{array}$ & $0.444 \pm 0.390$ & $0.452 \pm 0.390$ & $0.69(0.43-0.95)$ & $0.67(0.46-0.89)$ & $0.402 \pm 0.403$ & $0.360 \pm 0.399$ & $0.73(0.41-1.00)$ & $0.63(0.32-0.94)$ \\
\hline $\begin{array}{l}\text { Point, } \\
30 \text { min }\end{array}$ & $0.458 \pm 0.417$ & $0.457 \pm 0.406$ & $0.59(0.34-0.85)$ & $0.61(0.41-0.81)$ & $0.463 \pm 0.410$ & $0.458 \pm 0.394$ & $0.72(0.46-0.98)$ & $0.70(0.47-0.93)$ \\
\hline $\begin{array}{l}\text { Point, } \\
40 \text { min }\end{array}$ & $0.508 \pm 0.397$ & $0.504 \pm 0.388$ & $0.60(0.36-0.85)$ & $0.66(0.47-0.86)$ & $0.523 \pm 0.384$ & $0.508 \pm 0.371$ & $0.76(0.50-1.00)$ & $0.80 *(0.60-1.00)$ \\
\hline $\begin{array}{l}\text { Point, } \\
50 \text { min }\end{array}$ & $0.577 \pm 0.360$ & $0.567 \pm 0.405$ & $0.67(0.44-0.90)$ & $0.56(0.36-0.77)$ & $0.567 \pm 0.365$ & $0.577 \pm 0.395$ & $0.70(0.43-0.96)$ & $0.61(0.33-0.88)$ \\
\hline $\begin{array}{l}\text { Point, } \\
60 \text { min }\end{array}$ & $0.524 \pm 0.350$ & $0.500 \pm 0.391$ & $0.73(0.52-0.93)$ & $0.64(0.46-0.83)$ & $0.530 \pm 0.358$ & $0.364 \pm 0.565$ & $0.82^{*}(0.66-0.97)$ & $0.82^{*}(0.68-0.95)$ \\
\hline
\end{tabular}

${ }^{a} A U R O C$ : area under the receiver operating characteristic curve. *indicates good accuracy $(A U R O C \geq 0.80)$. 
TABLE 2: CESDA index for predicting infection and sepsis for worms found on points.

\begin{tabular}{|c|c|c|c|c|c|c|c|c|c|c|c|}
\hline \multicolumn{12}{|c|}{ Urine $<48$ hours $(n=45)$} \\
\hline $\begin{array}{l}\text { Infection } \\
\text { at } 40 \mathrm{~min}\end{array}$ & 0.232 & 0.80 & $\begin{array}{c}85 \\
(69-94)\end{array}$ & $\begin{array}{c}67 \\
(22-96)\end{array}$ & $\begin{array}{c}94 \\
(84-98)\end{array}$ & $\begin{array}{c}40 \\
(21-63)\end{array}$ & $\begin{array}{c}2.5 \\
(0.8-7.9)\end{array}$ & $\begin{array}{c}0.2 \\
(0.1-0.6)\end{array}$ & 82.2 & 0.40 & $0.016^{*}$ \\
\hline $\begin{array}{l}\text { Infection } \\
\text { at } 40 \mathrm{~min}\end{array}$ & 0.350 & 0.76 & $\begin{array}{c}72 \\
(53-86)\end{array}$ & $\begin{array}{c}75 \\
(19-99)\end{array}$ & $\begin{array}{c}95.8 \\
(81-99)\end{array}$ & $\begin{array}{c}25 \\
(13-42)\end{array}$ & $\begin{array}{c}2.9 \\
(0.5-16.0)\end{array}$ & $\begin{array}{c}0.4 \\
(0.2-0.8)\end{array}$ & 72.2 & 0.53 & 0.098 \\
\hline
\end{tabular}

${ }^{*} p \leq 0.05$ indicates significant association; CESDA: C. elegans Sepsis Detection Assay; AUROC: area under the receiver operating characteristic curve; Sn: Sensitivity; Sp: Specificity; PPV: Positive predictive value; NPV: Negative predictive value; PLR: Positive likelihood ratio; NLR: Negative likelihood ratio: Acc: Accuracy; $\mathbf{C l}$ : Confidence interval.

emission of olfactory molecules from the samples thus attracted the worms to gather at these points and increased the accuracy of the index in these points to predict both infection and septic urine samples. In addition, we scored the CESDA index of worms at 10-minute intervals for 60 minutes, compared with the NSDT protocol of observation at 60 minutes, and found that observation at 20 minutes provided a fair association of the index with sepsis and a good association with infection at 40 minutes. The attraction of the worms towards molecules emitted by cancer, sepsis or infection urine samples might require a different duration for worm migration; this observation might be useful for future experiments to validate the chemotaxis assay.

With the modifications performed in our study, CESDA has the potential to be developed into a simple assay which could screen for and predict both infection and sepsis in 20 minutes. The rapidity of this screening test could provide clinicians, and especially emergency medicine physicians, the results they need in a shorter time compared with the current diagnostic protocol, where serological tests and bacteriological cultures require 1-4 hours and more than 24 hours, respectively ${ }^{7}$. In addition, the chemotaxis assay uses urine as a diagnostic tool - this approach is beneficial especially for diagnosis in sepsis patients, as they are usually in hypovolemic shock where blood phlebotomy is complicated.

From the current analysis, we could not state for sure that those patients who were positive for the CESDA index in our study will not be diagnosed with cancer in the future; however, they were cancer-free when they were included in this study. As mentioned in the paper by Hirotsu et al., the olfactory molecule being sensed by the nematodes to detect cancer is still unknown ${ }^{9}$. As we did not perform olfactory neuron ablation on the nematodes used in this study, we cannot conclude from the results of this study whether the nematodes used in our study were attracted to the infected urine samples via olfactory cues. However, as cancer and infection share many T-cell-mediated inflammatory processes, it would be tempting to suggest that the worms might also be using their olfactory senses to detect olfactory molecules released because of activation of certain inflammatory processes in both infection and sepsis.

Due to the exploratory nature of this study, more samples need to be tested to validate further the nematodes' attraction towards urine samples from both infected and sepsis patients. Involvement of the nematodes' olfactory sense for their chemotaxis towards infected urine samples needs to be confirmed, and if verified, the associated olfactory molecule needs to be identified.

Acknowledgement: The authors would like to thank Dr Christabel Kang Wan Li, Dr Fatin Amirah bt Azmi, Dr Nadirah Hanim bt Abdu Hadi, Dr Dian Nasriana Nasuruddin and nurses of the UKMMC ED for their help in patient recruitment and funding procurement.

Conflict of interest: The authors declare that there is no conflict of interest.

Financial support: This study was funded by a Young Researcher Grant (GGPM-2013-102) from Universiti Kebangsaan Malaysia and a Fundamental Research Grant Scheme (FRGS/1/2014/SKK01/UKM/03/3) from Ministry of Higher Education, Malaysia awarded to Toh Leong Tan; and a grant from Ministry of Science, Technology \& Innovation (MOSTI) (SA1212001) 
awarded to Rahman Jamal. The funders had no role in the study design, data collection and analysis, decision to publish, or preparation of the manuscript.

\section{REFERENCES}

1. Singer M, Deutschman CS, Seymour CW, Shankar-Hari M, Annane $\mathrm{D}$, Bauer M, et al. The Third International Consensus Definitions for Sepsis and Septic Shock (Sepsis-3). JAMA. 2016;315(8):801-10.

2. Leitner E, Kessler HH, Spindelboeck W, Hoenigl M, Putz-Bankuti C, Stadlbauer-Kollner V, et al. Comparison of two molecular assays with conventional blood culture for diagnosis of sepsis. J Microbiol Methods. 2013;92(3):253-5.

3. Liu CL, Ai HW, Wang WP, Chen L, Hu HB, Ye T, et al. Comparison of 16S rRNA gene PCR and blood culture for diagnosis of neonatal sepsis. Arch Pediatr. 2014;21(2):162-9.

4. Seigel TA, Cocchi MN, Salciccioli J, Shapiro NI, Howell M, Tang A, et al. Inadequacy of temperature and white blood cell count in predicting bacteremia in patients with suspected infection. J Emerg Med. 2012;42(3):254-9.

5. Hisamuddin E, Hisam A, Wahid S, Raza G. Validity of C-reactive protein (CRP) for diagnosis of neonatal sepsis. Pak J Med Sci. 2015;31(3):527-31.

6. Markanday A. Acute Phase Reactants in Infections: EvidenceBased Review and a Guide for Clinicians. Open Forum Infect Dis. 2015;2(3):ofv098.
7. Tan TL, Ahmad NS, Nasuruddin DN, Ithnin A, Tajul Arifin K, Zaini IZ, et al. CD64 and Group II Secretory Phospholipase A2 (sPLA2-IIA) as Biomarkers for Distinguishing Adult Sepsis and Bacterial Infections in the Emergency Department. PLoS One. 2016;11(3):e0152065.

8. Goldstein B, King N. The Future of Cell Biology: Emerging Model Organisms. Trends Cell Biol. 2016;26(11):818-24.

9. Hirotsu $\mathrm{T}$, Sonoda $\mathrm{H}$, Uozumi $\mathrm{T}$, Shinden $\mathrm{Y}$, Mimori $\mathrm{K}$, Maehara Y, et al. A highly accurate inclusive cancer screening test using Caenorhabditis elegans scent detection. PLoS One. 2015;10(3):e0118699.

10. Fox AC, Robertson CM, Belt B, Clark AT, Chang KC, Leathersich $\mathrm{AM}$, et al. Cancer causes increased mortality and is associated with altered apoptosis in murine sepsis. Crit Care Med. 2010;38(3):88693.

11. Danai PA, Moss M, Mannino DM, Martin GS. The epidemiology of sepsis in patients with malignancy. Chest. 2006;129(6): 1432-40.

12. Hotchkiss RS, Moldawer LL. Parallels between cancer and infectious disease. N Engl J Med. 2014;371(4):380-3.

13. Levy MM, Fink MP, Marshall JC, Abraham E, Angus D, Cook D, et al. 2001 SCCM/ESICM/ACCP/ATS/SIS International Sepsis Definitions Conference. Intensive Care Med. 2003;29(4):530-8.

14. Yoshida K, Hirotsu T, Tagawa T, Oda S, Wakabayashi T, Iino Y, et al. Odour concentration-dependent olfactory preference change in C. elegans. Nat Commun. 2012;3:739. 